\title{
REVIEW
}

\section{Rapid response systems: are they really effective?}

\author{
Claudio Sandroni, Sonia D'Arrigo and Massimo Antonelli
}

\begin{abstract}
This article is one of ten reviews selected from the Annual Update in Intensive Care and Emergency Medicine 2015 and co-published as a series in Critical Care. Other articles in the series can be found online at http://ccforum.com/series/annualupdate2015. Further information about the Annual Update in Intensive Care and Emergency Medicine is available from http://www.springer.com/series/8901.
\end{abstract}

\section{Introduction}

Despite the immediate availability of qualified life support, the outcome of in-hospital cardiac arrest (IHCA) remains poor, with survival to discharge rarely exceeding $20 \%$ [1]. However, more than half of all cardiopulmonary arrests are preceded by deterioration in vital signs [2,3], which are often not appropriately evaluated, suggesting that many of these adverse events could be prevented by early identification and treatment [4].

In-hospital rapid response systems have been established to manage unstable patients in general wards, with the aim of preventing further deterioration leading to cardiac arrest [5]. Implementation of a rapid response system includes education of the ward staff (the afferent limb of the system) to systematically detect signs of physiological instability and identify patients needing urgent evaluation by a medical emergency team (MET). The MET (the efferent limb of the system) includes medical doctors and/or nurses experienced in management of critical patients; it is activated by ward staff in patients fulfilling specific criteria or in response to staff concerns, and its roles are to stabilize the patient in the ward or move the patient to a higher level of care.

Although the theory underlying MET systems is compelling, there is no definite evidence that their implementation improves patient outcome [6]. In effect, whereas a series of before-and-after, single center studies demonstrated benefit, the only multicenter randomized trial

\footnotetext{
* Correspondence: m.antonelli@rm.unicatt.it

Department of Anaesthesiology and Intensive Care, Catholic University of the Sacred Heart, A. Gemelli University Hospital, Rome, Italy
}

(RCT) conducted so far, the Medical Emergency Response, Intervention and Therapy (MERIT) study [7], did not show any significant change in the composite outcome of cardiac arrest, unexpected death, or unplanned intensive care unit (ICU) admission in the hospitals where MET was implemented. This and other results [6] have raised questions about the effectiveness of rapid response systems.

\section{Measures of effectiveness}

A key issue when evaluating the effectiveness of rapid response systems is the choice of the relevant outcome variables used for the measurement (Table 1). The main three outcome measures that have been employed are the rates of unexpected cardiac arrest, the rate of unplanned ICU admission, and the hospital mortality.

\section{Rates of unexpected cardiac arrest}

The earliest endpoint in the clinical course for a study addressing the effectiveness of rapid response systems is the rate of unexpected cardiac arrests occurring outside ICUs, i. e., the rates of cardiac arrest occurring in ward patients who have no do-not-attempt-resuscitation (DNAR) order.

Results of available studies almost consistently indicate that introduction of rapid response systems is associated with a significant reduction of in-hospital cardiac arrest rates (pooled risk ratio [RR] 0.64 [0.55-0.73]; p < 0.0001) (Figure 1). This endpoint, however, is potentially biased by the fact that part of the observed reduction in the rate of unexpected cardiac arrest observed after the implementation of a rapid response system may be due to a parallel increase in the fraction of expected cardiac arrests due to placement of a DNAR order. One of the tasks of METs in several institutions is to identify ward patients for whom resuscitation would be inappropriate. In these cases, calls to the MET result in discussion with the doctor who is in charge of the patient and in a decision to place a DNAR order or treatment limitations. For example, in a wellknown study from Buist et al. [8], the MET calls resulted in placement of a DNAR order for 17/124 patients (14\%), 13 of whom died. After the implementation of a rapid 
Table 1 Characteristics of interventional studies on rapid response systems

\begin{tabular}{|c|c|c|c|}
\hline Author, year & Country & Outcomes & Adjusted for \\
\hline Bristow, 2000 [13] & Australia & IHCA, hospital mortality, unplanned ICU admission & $C C, D$ \\
\hline Buist, 2002 [8] & Australia & IHCA, hospital mortality, unplanned ICU admission & $\mathrm{CM}$ \\
\hline Bellomo, 2003 [17] & Australia & IHCA, hospital mortality & no adj. \\
\hline DeVita, 2004 [37] & US & $\mathrm{IHCA}$ & $\mathrm{CM}$ \\
\hline Kenward, 2004 [9] & UK & IHCA, hospital mortality & no adj. \\
\hline Priestley, 2004 [38] & UK & Hospital mortality & $\mathrm{CM}, \mathrm{RCT}$ \\
\hline Hillman, 2005 [7] & Australia & IHCA, hospital mortality, unplanned ICU admission & $\mathrm{CM}, \mathrm{RCT}$ \\
\hline Jones, 2005 [29] & Australia & IHCA, hospital mortality & no adj. \\
\hline Dacey, 2007 [24] & US & IHCA, hospital mortality, unplanned ICU admission & no adj. \\
\hline Jolley, 2007 [26] & US & IHCA, hospital mortality & N/A \\
\hline Baxter, 2008 [25] & Canada & IHCA, hospital mortality, unplanned ICU admission & no adj. \\
\hline Chan, 2008 [11] & US & Non-ICU cardiac arrest, hospital mortality & $\mathrm{D}, \mathrm{CM}, \mathrm{T}$ \\
\hline Campello, 2009 [27] & Portugal & IHCA, hospital mortality & C, D \\
\hline Hatler, 2009 [30] & US & Non-ICU cardiac arrest & no adj. \\
\hline Konrad, 2010 [18] & Sweden & $\mathrm{IHCA}$ & C, D, LOS \\
\hline Lighthall, 2010 [28] & US & IHCA, hospital mortality & $\mathrm{S}, \mathrm{CCl}$ \\
\hline Santamaria, 2010 [19] & Australia & IHCA, hospital mortality, unplanned ICU admission & C, CM, D \\
\hline Beitler, 2011 [20] & US & IHCA, hospital mortality & $\mathrm{T}$ \\
\hline Laurens, 2011 [21] & Australia & IHCA, hospital mortality, unplanned ICU admission & no adj. \\
\hline Sarani, 2011 [31] & US & Non-ICU cardiac arrest, hospital mortality & no adj. \\
\hline Shah, 2011 [39] & US & IHCA, hospital mortality & $\mathrm{T}$ \\
\hline Howell, 2012 [40] & US & Hospital mortality & $\mathrm{CCl}, \mathrm{CM}, \mathrm{D}, \mathrm{S}, \mathrm{ICUBC}$ \\
\hline Simmes, 2012 [16] & The Netherlands & IHCA, unexpected mortality, unplanned ICU admission & $D, A S A$ \\
\hline Tobin, 2012 [22] & Australia & Hospital mortality & Year \\
\hline Al-Qahtani, 2013 [14] & Saudi Arabia & IHCA, hospital mortality & no adj. \\
\hline Chen, 2014 [23] & Australia & IHCA, hospital mortality & $D$, year \\
\hline
\end{tabular}

ASA: American Society of Anesthesiologists classification; C: comorbidities; CC: concurrent controls; CCI: Charlson Comorbidity Index; CM: case-mix severity; D: demographics; ICU: intensive care unit; ICUBC: intensive care unit bed capacity; IHCA: in-hospital cardiac arrest; LOS: length of stay; RCT: randomized clinical trial; S: seasonality; T: time trends; US: United States; UK: United Kingdom.

response system, the number of unexpected cardiac arrests decreased from 73 to 47 and the relevant mortality from $56 / 73(76.7 \%)$ to $26 / 47$ (55.3\%) ( $p=0.024)$. However, in the absence of a DNAR from the MET, the additional 13 deaths would have become unexpected cardiac arrests, potentially increasing mortality to $39 / 60$ ( $\mathrm{p}=0.2$ ). The percentage of MET interventions resulting in placement of a DNAR order is even higher in other studies, up to $24.6 \%$ [9] (Table 2).

\section{Rates of unplanned ICU admission}

Another endpoint for measuring rapid response system effectiveness is reduction in the rate of unplanned ICU admissions. The rationale is that the introduction of a rapid response system should increase the number of ICU admissions that are planned early, before further deterioration occurs, and decrease those occurring as emergencies, such as after resuscitation from cardiac arrest. This model has been indirectly investigated for ICU admissions from the emergency room [4], where an earlier transfer to the ICU has been demonstrated to decrease both ICU and hospital mortality. However, this is not always the case with rapid response systems. In a large American before-and-after study [11], in which 41.2\% of the MET interventions resulted in ICU admission, implementation of the rapid response system was followed by a significant reduction in non-ICU codes (from 6.08 pre-intervention to 3.08 post-intervention; $\mathrm{p}<0.001$ ) but this did not translate into a reduction in hospital-wide mortality rates per 100 admissions (from 3.22 pre-intervention to 3.09 post-intervention; $\mathrm{p}=0.41)$. In that study, mortality in patients transferred from ward to ICU was relatively high (43/155; $28 \%$ ), and issues in patient selection, appropriateness and timeliness of ICU transfer have been advocated to explain those results [12]. 


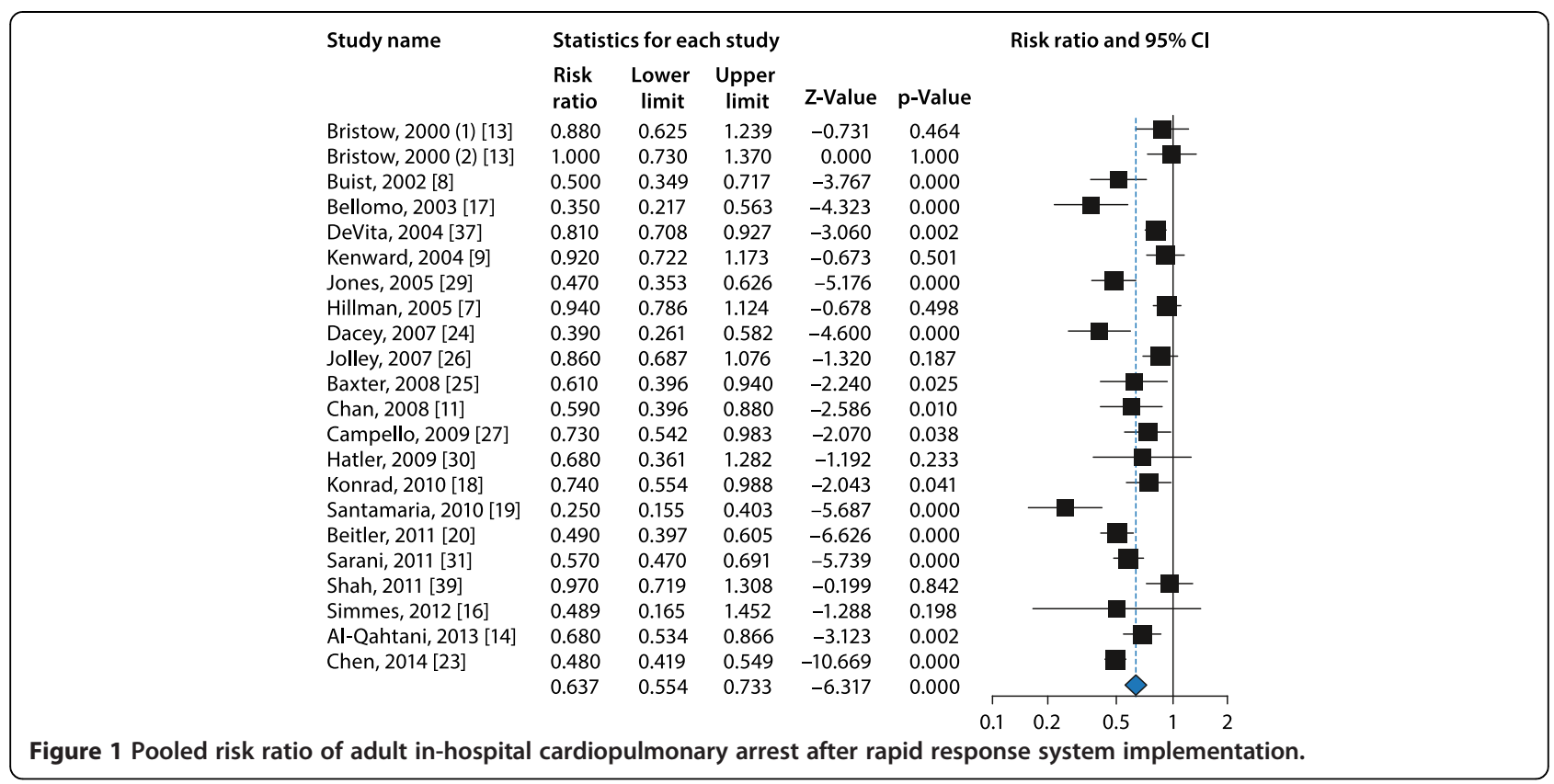

Moreover, although some studies demonstrated a decrease in the rate of unplanned ICU admissions [13,14] after the implementation of an in-hospital rapid response system, others $[7,15]$ showed no effect, and in a recent cohort study [16] rapid response system implementation was paradoxically followed by a significant increase in unplanned admissions to the ICU (from 34/1376 [2.47\%] to $100 / 2410$ [4.15\%]; OR 1.66, CI 1.07-2.55). Since the percentage of cardiac arrests in the study period decreased, although not significantly (from 4/1367 [0.29\%] to 3/2410 [0.12\%]; OR 0.38, CI 0.09-1.73), this result was explained by the authors as an increase in ICU referrals for unstable patients in the ward. However, it is worth noting that the

Table 2 The percentage of MET interventions resulting in placement of a DNAR order

\begin{tabular}{ll}
\hline Author, year & DNAR/MET calls (\%) \\
\hline Buist, 2002 [8] & $17 / 152(11.2)$ \\
Bellomo, 2003 [17] & $2 / 99(2)$ \\
Kenward, 2004 [9] & $32 / 130(24.6)$ \\
Hillman, 2005 [7] & $106 / 1319(8)$ \\
Jones, 2005 [29] & $18 / 327(5.5)$ \\
Chan, 2008 [11] & $73 / 376(19.4)$ \\
Lighthall, 2010 [28] & $10 / 378(2.6)$ \\
Beitler, 2011 [20] & $96 / 855(11.2)$ \\
Laurens, 2011 [21] & $55 / 296(18.5)$ \\
Al-Qahtani, 2013 [14] & $269 / 2879(9.3)$
\end{tabular}

On the other hand, placement of a DNAR order does not necessarily prevent MET intervention or the escalation of therapy. In a recent study [10], 45 ward patients with a DNR order in place were reviewed by the MET and $18(40 \%)$ were transferred to the ICU after MET activation. median APACHE II score at unplanned ICU admission was unchanged in that study, indicating that ICU referrals were apparently not done at an earlier stage of illness in the ward.

\section{Hospital mortality}

A reduction in hospital mortality represents the most comprehensive and important outcome measure for rapid response systems. In fact, the achievement of intermediate endpoints, such as the rates of unexpected cardiac arrests outside ICU or unplanned ICU admission, is of limited benefit, if the patient's final outcome does not change.

Results of earlier studies in terms of hospital mortality were conflicting, with several studies [8,14], [17-23] showing a reduction and others [9,11,13,24-28] showing no change or even an increase [29] in hospital mortality following rapid response system implementation. A systematic review and meta-analysis from Chan et al. [11] did not demonstrate any overall benefit from rapid response systems in terms of hospital mortality (pooled $\mathrm{RR}=0.96$ [0.84-1.09]) with a significant heterogeneity of results $\left(\mathrm{I}^{2}=90.3 \%\right.$; $\left.\mathrm{p}<0.001\right)$. However, a meta-analysis including more recent evidence showed an overall significant reduction in hospital mortality associated with the introduction of rapid response systems (pooled $R R=0.88$ [0.83-0.93] (Figure 2) even though heterogeneity remained significant $\left(\mathrm{I}^{2}=89.4 ; \mathrm{p}<0.001\right)$.

\section{Quality of evidence}

In general, the quality of evidence of observational studies on rapid response systems is low. Most of these 


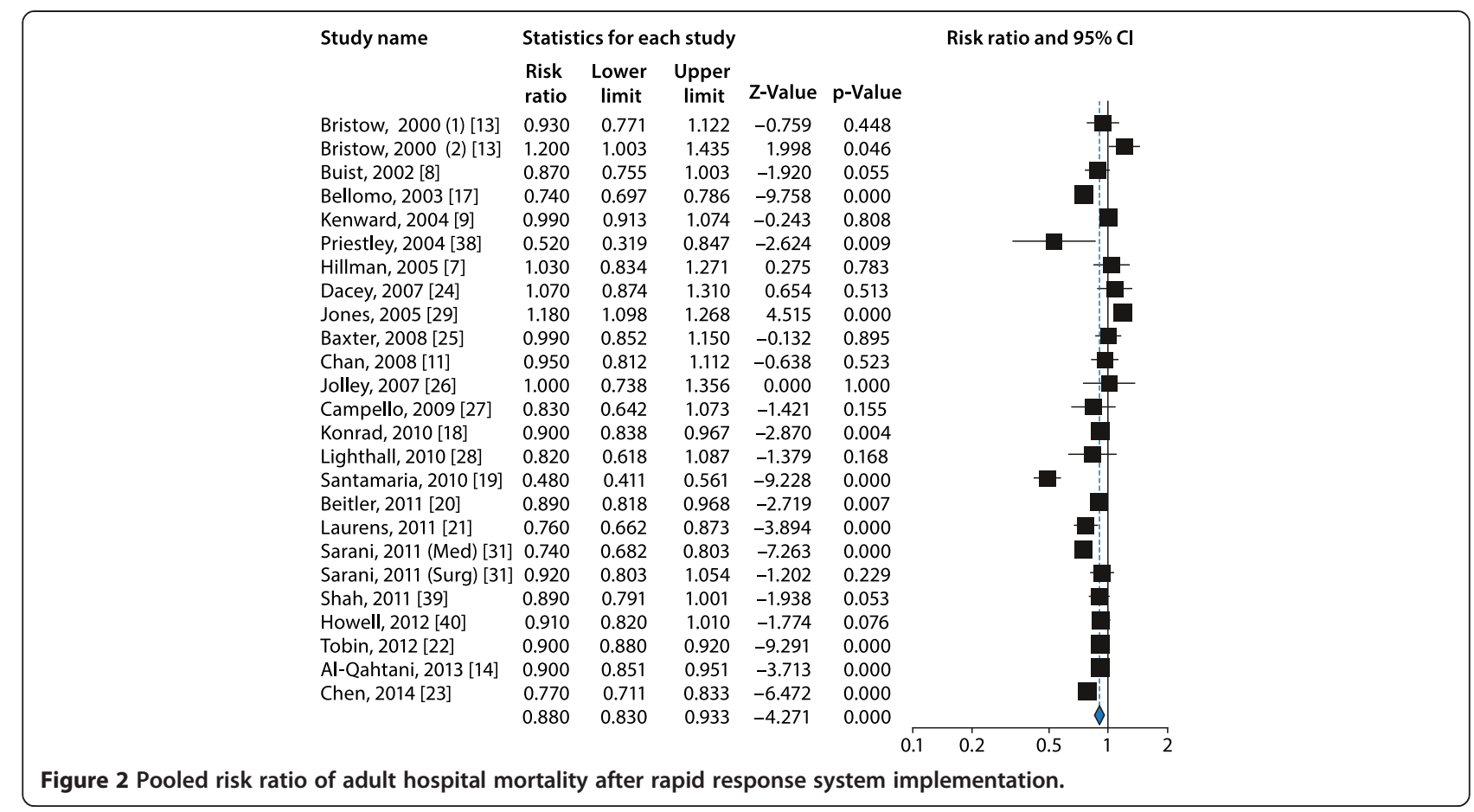

investigations assessed the in-hospital cardiac arrest rate without distinguishing the location of the event. Only a few studies $[8,11,14,20,24,30,31]$ limited the analysis to cardiac arrests outside the ICU and none of these studies conducted any blinded outcome assessment.

Almost all studies on rapid response systems have a before-and-after design, which makes them prone to bias. Indeed, in this kind of study, the reduction in hospital mortality observed during the study periods may be part of the secular trend started before the intervention and due to factors unrelated to rapid response system implementation, such as a change in the hospital case mix. In some of these studies, the adjustment for bias implicit in the study design has been made using multivariate models, including severity of illness or comorbidities, and autocorrelation (Table 1).

Randomized trials would represent the ideal solution to control for confounders in studies assessing rapid response system effectiveness. However, these trials have ethical and implementation issues. Patients cannot be randomized at individual level and cluster randomization should rather be used. On the other hand, in cluster randomization there is a high risk of contamination between the study arms because the study intervention cannot be blinded. This has been a major issue in the only multicenter randomized trial on rapid response systems conducted so far, the MERIT study [7], whose results were neutral. Although the authors of that study made every effort to prevent contamination, so that no specific training in the recognition of patients at risk had been made in hospitals in the control group, hospital safety issues in general and the benefits of the MET system in particular were largely reported in the media during the study period, which could have affected personnel behavior in the control hospitals. This is consistent with the fact that in the MERIT study the rates of in-hospital cardiac arrest decreased more in the hospitals of the control group than in those of the interventional group between the two study periods (from 2.61 to $1.64[\mathrm{p}=0.004]$ vs. 1.60 to $1.31[\mathrm{p}=0.171]$ ).

\section{Implementation issues}

Another major implementation issue in the MERIT trial, as in general for rapid response systems, was an afferent limb failure [32], i. e., absent or delayed MET activation by the ward staff in patients fulfilling MET calling criteria, due to an incomplete compliance of the ward personnel with the MET calling procedure. In the MERIT study, among 313 patients who had documented MET calling criteria more than 15 min before an unplanned ICU admission, the MET was actually called by the ward staff in only 95 cases (30\%). With such a low utilization rate, any potential benefit from the rapid response system would have been difficult to identify. Moreover, there is evidence that MET activation from the ward staff is often delayed, which is associated with increased hospital mortality $[33,34]$. Reasons for this afferent limb failure include adherence to the traditional system of calling the covering medical staff, a fear of creating false alarms, or disagreement with the MET calling criteria. Continuous education 
of the ward staff, review of the episodes of MET activation and feedback from MET users may help reduce those barriers and increase compliance with the rapid response system [19].

\section{Long-term effectiveness of rapid response systems}

The inconsistent results observed in studies on rapid response system effectiveness may also be due to insufficient time allowed for the evaluation of the study endpoint. One Australian study [19] showed that reduction in cardiac arrest rates achieved statistical significance at two years and reduction in hospital mortality at four years after the implementation of a rapid response system. Buist et al. [35] specifically examined rapid response system performance over time and found that the proportion of patients with delayed MET activation decreased as the rapid response system matured and that six years after MET implementation the MET calls had increased by $46 \%$ and the IHCA per 1,000 hospital admissions decreased by $24 \%$ a year (from 2.4 in 2000 to 0.66 in 2005). Similarly, a recent comparative study [36] showed that hospitals with mature rapid response systems performed better than similar hospitals where rapid response systems were recently implemented.

\section{Reproducibility}

A final issue for evaluating rapid response system effectiveness is reproducibility. The vast majority of studies is single-center and has been conducted in U.S. or Australian healthcare systems, while only a minority of studies has been conducted in the UK or in continental Europe. The effectiveness of a rapid response system depends on the nature and the quantity of the urgent, unmet patients' needs in general wards. This model may, therefore, not necessarily work in places where the severity of ward patients, the education of the ward personnel or the availability of human resources is different from those of places where this model was developed. International multicenter studies will be needed to ensure reproducibility of results.

\section{Conclusion}

There are different ways of measuring the effectiveness of rapid response systems. The major endpnoits include the rate of unexpected cardiac arrests outside the ICU, the rate of unplanned ICU admissions, and hospital mortality. All these outcome metrics have limitations and are prone to bias. The level of evidence supporting the effectiveness of rapid response systems is relatively low and almost all studies have a before-and-after design. Supporting evidence comes from a limited number of countries and needs to be reproduced in different hospital systems and organizations. Despite the ethical and implementation difficulties, high-quality randomized trials are warranted to reliably assess the effectiveness of rapid response systems.

\section{Abbreviations}

ASA: American society of anesthesiologists; CCl: Charlson comorbidity index DNAR: Do-not-attempt-resuscitation; ICU: Intensive care unit; ICUBC: Intensive care unit bed capacity; IHCA: In-hospital cardiac arrest; LOS: Length of stay; MET: Medical emergency team; RCT: Randomized clincial trial; RR: Risk ratio.

\section{Competing interests}

The authors declare that they have no competing interests.

\section{Declarations}

Publication of this article was funded by an independent research fund (\#8090110) from the Department of Anaesthesiology and Intensive Care - Catholic University School of Medicine, Rome, Italy.

Published online: 16 March 2015

\section{References}

1. Sandroni C, Nolan J, Cavallaro F, Antonelli M. In-hospital cardiac arrest: incidence, prognosis and possible measures to improve survival. Intensive Care Med. 2007;33:237-45.

2. Schein RM, Hazday N, Pena M, Ruben BH, Sprung CL. Clinical antecedents to in-hospital cardiopulmonary arrest. Chest. 1990;98:1388-92.

3. Hillman KM, Bristow PJ, Chey T, et al. Antecedents to hospital deaths. Intern Med J. 2001;31:343-8.

4. Chalfin DB, Trzeciak S, Likourezos A, Baumann BM, Dellinger RP. Impact of delayed transfer of critically ill patients from the emergency department to the intensive care unit. Crit Care Med. 2007;35:1477-83.

5. DeVita MA, Smith GB, Adam SK, et al. "Identifying the hospitalised patient in crisis" - a consensus conference on the afferent limb of rapid response systems. Resuscitation. 2010;81:375-82.

6. Chan PS, Jain R, Nallmothu BK, Berg RA, Sasson C. Rapid response teams: A systematic review and meta-analysis. Arch Intern Med. 2010;170:18-26.

7. Hillman K, Chen J, Cretikos M, et al. Introduction of the medical emergency team (MET) system: a cluster-randomised controlled trial. Lancet. 2005;365:2091-7.

8. Buist MD, Moore GE, Bernard SA, Waxman BP, Anderson JN, Nguyen TV. Effects of a medical emergency team on reduction of incidence of and mortality from unexpected cardiac arrests in hospital: preliminary study. BMJ. 2002;324:387-90.

9. Kenward G, Castle N, Hodgetts T, Shaikh L. Evaluation of a medical emergency team one year after implementation. Resuscitation. 2004;61:257-63.

10. Smith RL, Hayashi VN, Lee Yl, Navarro-Mariazeta L, Felner K. The medical emergency team call: a sentinel event that triggers goals of care discussion. Crit Care Med. 2014;42:322-7.

11. Chan PS, Khalid A, Longmore LS, Berg RA, Kosiborod M, Spertus JA. Hospital-wide code rates and mortality before and after implementation of a rapid response team. JAMA. 2008;300:2506-13.

12. Sherner $\mathrm{JH}$. Rapid response team implementation and hospital mortality rates. JAMA. 2009;301:1658-9.

13. Bristow PJ, Hillman KM, Chey T, et al. Rates of in-hospital arrests, deaths and intensive care admissions: the effect of a medical emergency team. Med J Aust. 2000;173:236-40.

14. Al-Qahtani S, Al-Dorzi HM, Tamim HM, et al. Impact of an intensivist-led multidisciplinary extended rapid response team on hospital-wide cardiopulmonary arrests and mortality. Crit Care Med. 2013;41:506-17.

15. Subbe CP, Davies RG, Williams E, Rutherford P, Gemmell L. Effect of introducing the Modified Early Warning score on clinical outcomes, cardio-pulmonary arrests and intensive care utilisation in acute medical admissions. Anaesthesia. 2003;58:797-802.

16. Simmes FM, Schoonhoven L, Mintjes J, Fikkers BG, van der Hoeven JG. Incidence of cardiac arrests and unexpected deaths in surgical patients before and after implementation of a rapid response system. Ann Intensive Care. 2012;2:20.

17. Bellomo R, Goldsmith D, Uchino S, et al. A prospective before-and-after trial of a medical emergency team. Med J Aust. 2003;179:283-7.

18. Konrad D, Jaderling G, Bell M, Granath F, Ekbom A, Martling CR. Reducing in-hospital cardiac arrests and hospital mortality by introducing a medical emergency team. Intensive Care Med. 2010;36:100-6. 
19. Santamaria J, Tobin A, Holmes J. Changing cardiac arrest and hospital mortality rates through a medical emergency team takes time and constant review. Crit Care Med. 2010;38:445-50.

20. Beitler JR, Link N, Bails DB, Hurdle K, Chong DH. Reduction in hospital-wide mortality after implementation of a rapid response team: a long-term cohort study. Crit Care. 2011;15:R269.

21. Laurens N, Dwyer T. The impact of medical emergency teams on ICU admission rates, cardiopulmonary arrests and mortality in a regional hospital. Resuscitation. 2011;82:707-12.

22. Tobin AE, Santamaria JD. Medical emergency teams are associated with reduced mortality across a major metropolitan health network after two years service: a retrospective study using government administrative data. Crit Care. 2012;16:R210

23. Chen J, Ou L, Hillman KM, et al. Cardiopulmonary arrest and mortality trends, and their association with rapid response system expansion. Med J Aust. 2014;201:167-70.

24. Dacey MJ, Mirza ER, Wilcox $V$, et al. The effect of a rapid response team on major clinical outcome measures in a community hospital. Crit Care Med. 2007:35:2076-82.

25. Baxter AD, Cardinal P, Hooper J, Patel R. Medical emergency teams at The Ottawa Hospital: the first two years. Can J Anaesth. 2008;55:223-31.

26. Jolley J, Bendyk H, Holaday B, Lombardozzi KA, Harmon C. Rapid response teams: do they make a difference? Dimens Crit Care Nurs. 2007;26:253-60.

27. Campello G, Granja C, Carvalho F, Dias C, Azevedo LF, Costa-Pereira A. Immediate and long-term impact of medical emergency teams on cardiac arrest prevalence and mortality: a plea for periodic basic life-support training programs. Crit Care Med. 2009;37:3054-61.

28. Lighthall GK, Parast LM, Rapoport L, Wagner TH. Introduction of a rapid response system at a United States veterans affairs hospital reduced cardiac arrests. Anesth Analg. 2010;111:679-86.

29. Jones D, Bellomo R, Bates $S$, et al. Long term effect of a medical emergency team on cardiac arrests in a teaching hospital. Crit Care. 2005;9:R808-15.

30. Hatler $C$, Mast $D$, Bedker $D$, et al. Implementing a rapid response team to decrease emergencies outside the ICU: one hospital's experience. Medsurg Nurs. 2009:18:84-90

31. Sarani B, Palilonis E, Sonnad S, et al. Clinical emergencies and outcomes in patients admitted to a surgical versus medical service. Resuscitation. 2011:82:415-8

32. Sandroni C, Cavallaro F. Failure of the afferent limb: a persistent problem in rapid response systems. Resuscitation. 2011;82:797-8.

33. Trinkle RM, Flabouris A. Documenting Rapid Response System afferent limb failure and associated patient outcomes. Resuscitation. 2011;82:810-4.

34. Calzavacca P, Licari E, Tee A, et al. The impact of Rapid Response System on delayed emergency team activation patient characteristics and outcomes - a follow-up study. Resuscitation. 2010;81:31-5.

35. Buist M, Harrison J, Abaloz E, Van Dyke S. Six year audit of cardiac arrests and medical emergency team calls in an Australian outer metropolitan teaching hospital. BMJ. 2007:335:1210-2.

36. Chen J, Ou L, Hillman K, et al. The impact of implementing a rapid response system: A comparison of cardiopulmonary arrests and mortality among four teaching hospitals in Australia. Resuscitation. 2014;85:1275-81.

37. DeVita MA, Braithwaite RS, Mahidhara R, Stuart S, Foraida M, Simmons RL. Use of medical emergency team responses to reduce hospital cardiopulmonary arrests. Qual Saf Health Care. 2004;13:251-4.

38. Priestley G, Watson W, Rashidian A, et al. Introducing Critical Care Outreach: a ward-randomised trial of phased introduction in a general hospital. Intensive Care Med. 2004;30:1398-404.

39. Shah SK, Cardenas Jr VJ, Kuo YF, Sharma G. Rapid response team in an academic institution: does it make a difference? Chest. 2011;139:1361-7.

40. Howell MD, Ngo L, Folcarelli P, et al. Substained effectiveness of a primary-team-based rapid response system. Crit Care Med. 2012;40:2562-8. 